\title{
The effect of physician prescribing patterns based on ESC guidelines on morbidity improvement among heart failure patients
}

\author{
Ema Pristi Yunita ${ }^{* 1,2}$, Anindhita Dwi Safitri ${ }^{1}$, Ardian Rizal ${ }^{3,4}$ \\ ${ }^{1}$ Department of Pharmacy, Faculty of Medicine, Universitas Brawijaya, \\ $J$. Veteran, Malang, Indonesia \\ ${ }^{2}$ Research Center of Smart Molecules of Natural Genetic Resources (SMONAGENES), Universita Brawijaya, \\ $\mathrm{Jl}$. Veteran, Malang, Indonesia \\ ${ }^{3}$ Department of Cardiology, General Hospital of Dr. Saiful Anwar, \\ Jl. Jaksa Agung Suprapto, Malang, Indonesia \\ ${ }^{4}$ Department of Cardiology, Islamic Hospital of Aisyiyah, \\ Jl. Sulawesi, Malang, Indonesia
}

Submitted: 18-05-2020

Reviewed: 20-01-2021

Accepted: 16-03-2021

\begin{abstract}
Data from Basic Health Research shows the prevalence of heart failure in Indonesia is between $0.1 \%$ to $0.3 \%$ of the entire Indonesian population. This number is predicted to increase from year to year. Heart failure is a complex syndrome that can cause abnormalities in the structure and function of the heart. Based on the ejection fraction values, there are three types of heart failure, namely $\mathrm{HFrEF}$ (EF: < 40\%), HFmrEF (EF: 40-49\%), and HFpEF (EF: $\geq 50 \%$ ). Considering that the type of heart failure requiring treatment according to ESC guidelines is only HFrEF, however, this study would also evaluate the effect of physician prescribing patterns on the morbidity of HFmrEF. The recommendations from ESC guidelines to the treatment of HFrEF that can reduce morbidity and mortality are three medication combinations, namely ACE inhibitors/angiotensin receptor blockers, beta-blockers, and aldosterone antagonists. Therefore, this study aims to determine the effect of the suitability of physician prescribing patterns according to ESC guidelines on improving the morbidity of heart failure patients in HFrEF and HFmrEF type. Improvements in morbidity can be seen from the quality of life score and frequency of hospitalization by using questionnaires. The study was conducted in the cardiology outpatient clinic of Dr. Saiful Anwar General Hospital and Islamic Hospital of Aisyiyah in April-May 2019. Subjects who participated in the study were 57 patients. The One-way ANOVA test results showed no significant difference between physician prescribing patterns of quality of life scores on HFrEF ( $p=0.944)$ while the Kruskal Wallis test for the same parameters on HFmrEF also showed insignificant results $(\mathrm{p}=0.210)$. The Kruskal Wallis test results showed no significant difference between the patterns of physician prescribing to the frequency of hospitalization in both HFrEF and HFmrEF ( $p=0.260 ; p=0.428$ ). The results showed that physician prescribing patterns in accordance with ESC guidelines resulted in the best quality of life scores on HFrEF. The lowest frequency of hospitalization was also shown in HFrEF patients who received treatment according to ESC guidelines.
\end{abstract}

Keywords: Ejection fraction, heart failure, ESC guidelines, physician prescribing patterns

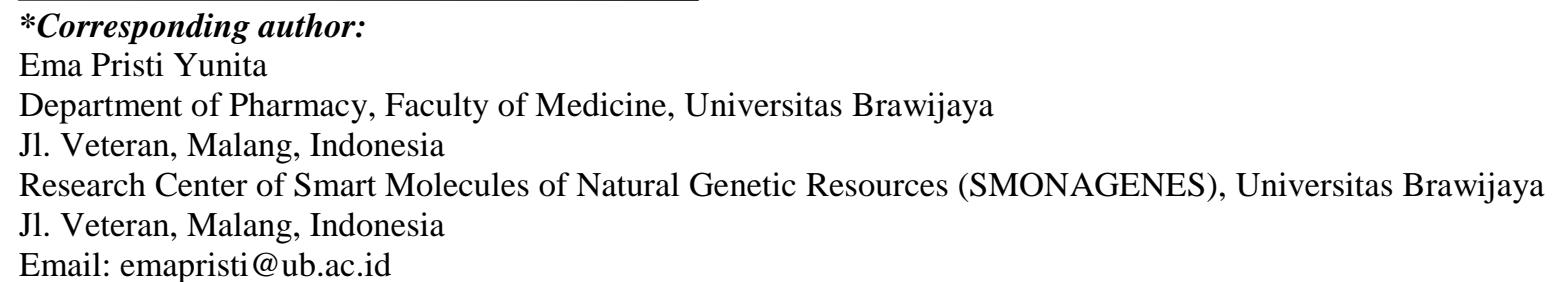




\section{INTRODUCTION}

As many as 17.9 million people $(31 \%)$ of 58 million deaths in the world are caused by cardiovascular disease (WHO, 2020). Indonesia occupies the second-highest rank in Southeast Asia with a total of 371 thousand cardiovascular patients (WHO, 2007). Heart failure can be caused by an abnormal structure and function of heart condition that causes a decrease in heart-pumping capacity thereby reducing ventricular filling and contraction of the myocardium. In the case of myocardial infarction, chronic hypertension, and cardiomyopathy, the heart undergoes cell remodeling through a variety of complex biochemical mechanisms (Mcmurray et al., 2012).

The remodeling process in the initial phase can improve the necrosis area and the formation of scar tissue so that there is no expansion of infarction. However, the amount of remodeling is proportional to the size of the infarction. After infarction occurs, the area of injury soon expands, followed by regional dilation and thinning infarction zone. This situation causes changes in ventricular mass, composition, and volume that can affect the heart function (Kehat and Molkentin, 2010).

The results of the meta-analysis showed that cardiac medications that can cause a decrease in mortality and morbidity are a combination of ACE inhibitors, beta-blockers, and aldosterone antagonists (56\%); monotherapy ACE inhibitor (16\%); and monotherapy angiotensin receptor blockers (12\%) (Burnett et al., 2017). This finding refers to the European Society of Cardiology (ESC) guidelines that the use of a combination of the three medications, that are ACE inhibitors, beta-blockers, and aldosterone antagonists, can increase the highest survival. Therefore, a combination of the three medications is recommended for patients with HFrEF (Heart Failure with reduced Ejection Fraction) except those who are intolerant of these medications (Ponikowski et al., 2016).

The therapeutic error can be influenced by several factors, namely errors in medications selection, dosage, method of use, and illegible writing (Oshikoya et al., 2009). According to data on prescribing patterns for heart failure medications in Asia, the most common pattern of using heart failure medications in Indonesia is ACE inhibitors/angiotensin receptor blockers (87\%), and the least is betablockers (61\%) (Teng et al., 2018). Therefore, a current alignment of patient therapy to the guidance is crucial for the management of heart failure patients.

The basic goals of heart failure therapy include improving symptoms, increasing functional capacity, improving quality of life, decreasing hospitalization frequency, and decreasing mortality rates (Berliner and Bauersachs, 2017). Consequently, this study focuses on analyzing the differences in the morbidity of patients with HFrEF and HFmrEF (Heart Failure with mid-ranged Ejection Fraction) on the suitability of physician prescribing patterns according to ESC guidelines. The parameters used in this study to assess the improvement in morbidity are increasing the quality of life scores and decreasing the frequency of hospitalization.

\section{MATERIALS AND METHODS}

The design of this study was an observational analytic with a cross-sectional study approach. The study used medical records and modified MLHF (Minnesota Living with Heart Failure) questionnaires. This study compared the improvement in the morbidity of patients who received appropriate and inappropriate prescribing patterns that refer to ESC guidelines. Based on the calculation of the sample size formula, it is known that the minimum sample size in this study was 51 patients. To obtain the sample size, a two-month study was conducted, from April to May 2019.

The study inclusion criteria were adult patients aged $>25$ years with a diagnosis of heart failure, have received heart failure medications and have taken routine medications for at least the last three months, had an ejection fraction value $<50 \%$, and were willing to participate in the study. The study was conducted at Dr. Saiful Anwar General Hospital and Islamic Hospital of Aisyiyah in Malang. This research methodology has obtained ethical clearance from the Health Research Ethics Commission Dr. Saiful Anwar General Hospital Malang through decree number 400/048/K.3/302/2019. 
The morbidity assessment on the presence or absence of significant differences in the quality of life scores between medication groups in HFrEF patients was using the One-way ANOVA, while in HFmrEF patients was using with the Kruskal Wallis. Morbidity assessment on the presence or absence of significant differences in the frequency of repeated hospitalizations within the last six months between medication groups in HFrEF and HFmrEF patients was using the Kruskal Wallis. The p-value was significant if $<0.05$ at $95 \%$ confidence interval.

\section{RESULT AND DISCUSSION}

This study obtained 57 HFrEF and HFmrEF patients that met the inclusion and exclusion criteria. Based on the ejection fraction value, there were three types of heart failure, namely HFrEF (Heart Failure with reduced Ejection Fraction) (EF: < 40\%), HFmrEF (Heart Failure with mid-ranged Ejection Fraction) (EF: 40-49\%), and HFpEF (Heart Failure with preserved Ejection Fraction) (EF: $\geq 50 \%$ ) (Ponikowski et al., 2016). Patient characteristics were based on age group, gender, and comorbid diseases as seen in Table 1 . The elderly group $(54.4 \%)$ is the age group that experience heart failure more because the prevalence of heart failure increases with age which is around $0.5 \%$ at the age of 20 39 years, and the prevalence will continue to increase to more than $10 \%$ at the age of 80 years and over. This is due to aging factors in the structure and function of the heart including increased arterial and heart muscle stiffness, decreased relaxation of the diastolic heart muscle, and an increase in left ventricular mass (Upadhya and Kitzman, 2017). Based on gender, this study showed that patients who had more heart failure were men $(77.2 \%)$ than women $(22.8 \%)$. This refers to the latest results from the Framingham Heart Study over the past 40 years that the cases of heart failure in women have decreased by about one third while in men there is no change in the prevalence of heart failure (Parker et al., 2008). The results of the Framingham Heart Study show that the etiology of heart failure can be sorted hierarchically into Coronary Heart Disease (CHD), valvular heart disease, hypertension, and other etiologies/unknown (Lee et al., 2009). The percentage hierarchy of comorbidities in this study ranging from the largest to the lowest were CHD (35.0\%), unknown (26.3\%), Diabetes Mellitus (DM) + CHD (19.3\%), hypertension + CHD (8.8\%), DM (5.3\%), hypertension + DM (1.8\%), hypertension + DM + CHD (1.8\%), and hypertension (1.7\%).

Table 1. Characteristics of HFrEF and HFmrEF patients

\begin{tabular}{lc}
\hline \multicolumn{1}{c}{ Characteristics } & Number of Patients (\%) \\
\hline Age & $26(45.6)$ \\
26-55 years old & $31(54.4)$ \\
$>$ 55 years old & $\mathbf{5 7 ( 1 0 0 . 0 )}$ \\
\hline Total & $44(77.2)$ \\
\hline Gender & $13(22.8)$ \\
Male & $\mathbf{5 7 ( 1 0 0 . 0 )}$ \\
Female & \\
\hline Total & $20(35.0)$ \\
\hline Comorbid Diseases & $15(26.3)$ \\
Coronary Heart Disease (CHD) & $11(19.3)$ \\
Unknown & $5(8.8)$ \\
DM + CHD & $3(5.3)$ \\
Hypertension + CHD & $1(1.8)$ \\
Diabetes Mellitus (DM) & $1(1.8)$ \\
Hypertension & $1(1.8)$ \\
Hypertension + DM & $\mathbf{5 7}(\mathbf{1 0 0 . 0})$ \\
\hline Hypertension + DM + CHD & \\
\hline Total &
\end{tabular}


Based on the results of the study (Table 2), the most prescribed medication for heart failure by physician for HFrEF and HFmrEF patients was a combination of three medications, i.e. ACE inhibitors/angiotensin receptor blockers, beta-blockers (bisoprolol), and aldosterone antagonists (spironolactone), which was 25 patients (43.9\%). This was in accordance with ESC guidelines for the treatment of HFrEF patients but the therapy management for HFmrEF patients does not have to use the combination of these three medications (Ponikowski et al., 2016). Therefore, the definition of therapy according to ESC guidelines was that if HFrEF patients get a combination of the three medications $(58.1 \%)$ and the therapy was said to be inappropriate if the physician prescribes it outside of the combination of the three medications, i.e. monotherapy ACE-I/ARB (3.2\%), ACE-I/ARB + Bisoprolol $(19.4 \%)$, or ACE-I/ARB + Spironolactone $(19.4 \%)$. Meanwhile, all HFmrEF patients have received appropriate treatment because according to ESC guidelines there was no specific treatment that shows a decrease in morbidity or mortality in patients with HFpEF or HFmrEF. However, these HFpEF or HFmrEF patients were mostly among the elderly, very symptomatic, and often have a poor quality of life, so the important goal of therapy for this group of patients was to reduce the symptoms and improve health (Ponikowski et al., 2016).

Table 2. Physician prescribing patterns for HFrEF and HFmrEF patients

\begin{tabular}{lclc}
\hline \multicolumn{1}{c}{$\begin{array}{c}\text { Suitability of } \\
\text { HFrEF Therapy }\end{array}$} & $\begin{array}{c}\text { Number of Patients } \\
(\boldsymbol{\%})\end{array}$ & \multicolumn{1}{c}{$\begin{array}{c}\text { Suitability of } \\
\text { HFmrEF Therapy }\end{array}$} & $\begin{array}{c}\text { Number of } \\
\text { Patients (\%) }\end{array}$ \\
\hline Appropriate & $\mathbf{1 8 ( 5 8 . 1 )}$ & Appropriate & $\mathbf{2 6}(\mathbf{1 0 0 . 0})$ \\
ACE-I/ARB + Biso + & $18(58.1)$ & ACE-I/ARB + Biso & $12(46.2)$ \\
Spiro & $\mathbf{1 3 ( 4 1 . 9 )}$ & ACE-I/ARB + Biso + Spiro & $7(26.9)$ \\
Inappropriate & $6(19.4)$ & ACE-I/ARB + Spiro & $4(15.4)$ \\
ACE-I/ARB + Biso & $6(19.4)$ & Biso single & $1(3.8)$ \\
ACE-I/ARB + Spiro & $1(3.2)$ & Biso + Spiro & $1(3.8)$ \\
ACE-I/ARB single & $\mathbf{3 1 ( \mathbf { 1 0 0 . 0 } )}$ & Total & $1(3.8)$ \\
\hline Total & & & $\mathbf{2 6 ( 1 0 0 . 0 )}$ \\
\hline
\end{tabular}

Notes:

ACE-I = Angiotensin Converting Enzyme Inhibitor; ARB = Angiotensin Receptor Blocker; Biso = Bisoprolol; Spiro $=$ Spironolactone

The success of heart failure treatment in this study was measured through two parameters, namely the assessment of the patients' quality of life and the frequency of repeated hospitalizations in the hospital in the past six months. Quality of life related to Health-related Quality of Life (HQoL) includes functional limitations that are physical, mental, and spiritual. HQoL can be used as an integrative measure that brings together mortality and morbidity (Syahidah, 2017). Symptoms of heart failure such as shortness of breath and fatigue can be related to patients' physical activity limitations and physiological pressure which can worsen their health related to HQoL. Therefore, HQoL was used as a predictor of morbidity for heart failure patients which can be in a form of measurement of the results of intervention evaluation and predictions of hospitalization events and mortality (Tangsatitkiat and Sakthong, 2010). The quality of life of heart failure patients can be affected by several factors, such as age, ejection fraction, medications use, patient compliance, and economic level (Yaghoubi et al., 2012).

Quality of life contains two components, the subjective component or people's perception and the objective component. In this study, the subjective component was used to assess the quality of life of heart failure patients through a set of questions or questionnaires. The answers to these questions were then changed to values or scales so that they can be measured objectively (Brink et al., 2009). In general, HQoL can be measured by two approaches, i.e. general and specific instruments (Rascati, 2014). Specific instruments for heart disease include Minnesota Living with Heart Failure Questionnaire 
(MLHFQ), Chronic Heart failure Questionnaire (CHQ), and Kansas City Cardiomyopathy Questionnaire (KCCQ). MLHFQ is the most widely used questionnaire as a disease-specific instrument for assessing the clinical condition of heart failure patients and has been used comprehensively to assess the perception of the effects of heart failure and its treatment on the daily lives of patients (Tangsatitkiat and Sakthong, 2010). This study used a modified MLHFQ questionnaire instrument so that it can assess the quality of life of HFrEF and HFmrEF patients. Interpretations of quality of life scores in this study were good quality of life (score $\leq 19$ ), moderate quality of life (score 20-66), and poor quality of life (score $\geq 67$ ).

Validity and reliability test were conducted before the MLHFQ questionnaire was used to assess the HQoL of research subjects. The tests were carried out on five patients because the large sample requirements for the validity and reliability test is $10 \%$ of the total sample. The distribution of the $\mathrm{r}$ table with a significance value of $5 \%$ for five patients was 0.878 . Therefore, Pearson correlation values on each question item that has a value $>0.878$ can be declared valid and can be continued with the reliability test. In this study, from a total of 21 question items, it was found that only 17 items had a Pearson correlation value $>0.878$ (valid). Furthermore, a reliability test of the 17 question items was conducted. The research questionnaire was declared reliable because the value of Cronbach's alpha > alpha coefficient 0.6 was 0.987 .

The morbidity parameter consisted of quality of life scores and frequency of hospitalization. Hypothesis testing conducted was a comparative analysis to find out the comparison in the difference between physician prescribing patterns to the quality of life scores of patients with HFrEF and HFmrEF ( $p=0.944 ; p=0.210$ ) and the frequency of repeated hospitalization of HFrEF and HFmrEF patients ( $p$ $=0.260 ; p=0.428$ ) at heart failure patients. The data can be seen in Table 3. Significance value for each parameter obtained was $>0.05$ which means there was no statistically significant difference due to the disproportionate sample size between medication groups. The small sample size between the medication groups had caused no statistically apparent difference in morbidity parameters. However, the overall total sample obtained, as many as 57 patients, had met the minimum sample size requirements in this study. However, the results showed that the use of prescription medications according to ESC guidelines (combination of 3 drugs) had the best assessment of morbidity conditions, i.e. the highest level of quality of life (score 25.81 included in the category of moderate quality of life) and the lowest frequency of hospitalization (score 1.00) in HFrEF patients. This was because HFrEF patients were known to benefit from a reduction in the morbidity rate from the use of the latest ESC guidance algorithm (Ponikowski et al., 2016). ACE (angiotensin-converting enzyme) inhibitors, angiotensin receptor blockers, and aldosterone antagonists act on the inhibition of the Renin-AngiotensinAldosterone system that can overcome excessive activation in patients with heart failure (Martin et al., 2018). Decreased sympathetic activity and decreased aldosterone and vasopressin can cause a decrease in arterial and venous pressure which can reduce preload and afterload. This can affect the increase in stroke volume and improve the ejection fraction. In addition, these medications also work as antiremodeling by inhibiting myocardial hypertrophy and apoptosis in cardiomyocytes due to decreased neurohormonal activation, i.e. decreased levels of Angiotensin II, aldosterone, and norepinephrine (Bratsos, 2019). 
Table 3. The morbidity parameters of HFrEF and HFmrEF patients according to the therapy group

\begin{tabular}{lcccccc}
\hline \multicolumn{1}{c}{ Parameters } & HFrEF & N & $\mathbf{p}$ & $\begin{array}{c}\text { HFmr } \\
\text { EF }\end{array}$ & N & p \\
\hline Quality of Life Mean Scores & & & & & & \\
Monotherapy Biso & 0 & 0 & & 25.00 & 1 & \\
Monotherapy ACE-I/ARB & 28.00 & 1 & & 14.00 & 1 & \\
Biso + Spiro & 0 & 0 & $0.944^{\mathrm{a}}$ & 48.00 & 1 & 0.210 \\
ACE-I/ARB + Biso & 29.00 & 7 & & 30.10 & 11 & $\mathrm{~b}$ \\
ACE-I/ARB + Spiro & 29.83 & 6 & & 33.00 & 4 & \\
ACE-I/ARB + Biso + Spiro & 25.81 & 16 & & 24.00 & 9 & \\
\hline Total & & 30 & & & 27 & \\
\hline Frequency of Repeated Hospitalizations & & & & & & \\
Monotherapy Biso & 0 & 0 & & N/A & 1 & \\
Monotherapy ACE-I/ARB & N/A & 1 & & N/A & 1 & \\
Biso + Spiro & 0 & 0 & $0.260^{\mathrm{b}}$ & N/A & 1 & 0.428 \\
ACE-I/ARB + Biso & 1.75 & 7 & & 1.67 & 11 & $\mathrm{~b}$ \\
ACE-I/ARB + Spiro & 1.60 & 6 & & 1.00 & 4 & \\
ACE-I/ARB + Biso + Spiro & 1.00 & 16 & & 1.00 & 9 & \\
\hline Total & & 30 & & & 27 & \\
\hline
\end{tabular}

\section{Notes:}

N/A: not available

${ }^{\mathrm{a}}$ : The hypothesis test used is One-way ANOVA

${ }^{\mathrm{b}}$ : The hypothesis test used is Kruskal Wallis p-value is statistically significant if $<0.05$ at the $95 \%$ confidence interval

ACE-I = Angiotensin Converting Enzyme Inhibitor; ARB = Angiotensin Receptor Blocker; Biso = Bisoprolol; Spiro $=$ Spironolactone

In HFmrEF patients, they have the same lowest frequency of hospitalization for both the combination of three medications (score 1.00) and the combination of ACE inhibitors/angiotensin receptor inhibitors + spironolactone (score 1.00). The highest level of quality of life was in patients who get monotherapy ACE inhibitors/angiotensin receptor blockers (score 14.00 included in the category of good quality of life). According to the latest ESC guideline therapy algorithm, there was no specific treatment that has been proven to show a decrease in morbidity in HFmrEF patients, so the treatments chosen are those that relieve the symptoms felt by the patient (Ponikowski et al., 2016).

The frequency of repeated hospitalizations in HFrEF and HFmrEF patients that obtained a combination of three drugs according to the ESC guidelines in this study was quite good because patients only experienced once repeated hospitalization in the last six months. This shows that the patient did not experience a recurrence of episodes of heart failure (Majid, 2010). Based on Ogbemudia and Asekhame (2016), the highest rate of repeat admission was at the age > 65 years, which was about more than $50 \%$ of patients were retreated within six months after the hospital discharge (Ogbemudia and Asekhame, 2016). However, this did not occur in patients in this study whose majority were $>55$ years old. This can be caused by several factors including the patients obtained good health education about how to take care for themselves at home, the patients used medications appropriately, there was good communication from health care providers to patients, and there was a good follow-up planning after hospital discharge (Majid, 2010). 


\section{CONCLUSION}

Based on the results of the study, it was found that the use of prescribing patterns according to ESC guidelines, that is a combination of three medications (ACE inhibitors/angiotensin receptor blockers, bisoprolol, and spironolactone), is the most commonly used for HFrEF and HFmrEF patients. In addition, HFrEF patients who have the highest level of quality of life and experience the lowest frequency of hospitalization are patients in the group given prescribing pattern according to ESC guidelines.

\section{ACKNOWLEDGEMENT}

The researcher would like to thank the Faculty of Medicine Universitas Brawijaya (FMUB) for funding this research from the 2017 FMUB PNBP with the Contract Number: 19/SK/UN10.7/PN/BPPM/2017.

\section{REFERENCES}

Berliner, D., \& Bauersachs, J. (2017). Current drug therapy in chronic heart failure - the new guidelines of the european society of cardiology (ESC). Korean Circulation Journal, 47(5), 543-554. https://doi.org/10.4070/kcj.2017.0030

Bratsos, S. (2019). Efficacy of angiotensin converting enzyme inhibitors and angiotensin receptorneprilysin inhibitors in the treatment of chronic heart failure: a review of landmark trials. Cureus, 11(1), 1-8. https://doi.org/10.7759/cureus.3913

Brink, E., Persson, L. O., \& Karlson, B. W. (2009). Coping with myocardial infarction: Evaluation of a coping questionnaire. Scandinavian Journal of Caring Sciences, 23(4), 792-800. https://doi.org/10.1111/j.1471-6712.2008.00666.x

Burnett, H., Earley, A., Voors, A. A., Senni, M., McMurray, J. J. V., Deschaseaux, C., \& Cope, S. (2017). Thirty years of evidence on the efficacy of drug treatments for chronic heart failure with reduced ejection fraction: a network meta-analysis. Circulation Heart Failure, 10, 1-13. https://doi.org/10.1161/CIRCHEARTFAILURE.116.003529

Kehat, I., \& Molkentin, J. D. (2010). Molecular pathways underlying cardiac remodeling during $\begin{array}{llll}\text { pathophysiologic } & \text { stimulation. } & \text { Circulation, }\end{array}$ https://doi.org/10.1161/CIRCULATIONAHA.110.956839

Lee, D. S., Gona, P., Vasan, R. S., Larson, M. G., Benjamin, E. J., Wang, T. J., Tu, J. V., \& Levy, D. (2009). Relation of disease etiology and risk factors to heart failure with preserved or reduced ejection fraction: insights from the national heart, lung, and blood institutes's framingham heart study. $\quad$ Circulation, 119(24), 3070-3077. https://doi.org/10.1161/CIRCULATIONAHA.108.815944.RELATION

Majid, A. (2010). Factors analysis felated to readmission on patient with congestive heart failure in Yogyakarta Hospital year 2010. University of Indonesia.

Martin, N., Manoharan, K., Thomas, J., Davies, C., \& Lumbers, R. T. (2018). Beta-blockers and inhibitors of the renin-angiotensin aldosterone system for chronic heart failure with preserved ejection fraction. Cochrane Database of Systematic Reviews, 2018(6), 1-197. https://doi.org/10.1002/14651858.CD012721.pub2

Mcmurray, J. J. V, Adamopoulos, S., Anker, S. D., Auricchio, A., Bohm, M., Dickstein, K., Falk, V., Filippatos, G., Fonseca, C., Gomez-Sanchez, M. A., Jaarsma, T., Køber, L., Lip, G. Y. H. ., Maggioni, A. Pietro, Parkhomenko, A., Pieske, B. M., Popescu, B. A., Rønnevik, P. K., Rutten, F. H., ... Zeiher, A. (2012). ESC Guideines for the diagnosis and treatment of acute and chronic heart failure 2012: The Task Force for the Diagnosis and Treatment of Acute and Chronic Heart Failure 2012 of the European Society of Cardiology. European Heart Journal, 33, 1787-1847. https://doi.org/10.1093/eurheartj/ehs104

Ogbemudia, E. J., \& Asekhame, J. (2016). Rehospitalization for heart failure in the elderly. Saudi Medical Journal, 37(10), 1144-1147. https://doi.org/10.15537/smj.2016.10.15259 
Oshikoya, K. A., Senbanjo, I. O., \& Amole, O. O. (2009). Interns’ knowledge of clinical pharmacology and therapeutics after undergraduate and on-going internship training in Nigeria: a pilot study. BMC Medical Education, 9(50), 1-9. https://doi.org/10.1186/1472-6920-9-50

Parker, R. B., Rodgers, J. E., \& Cavallari, L. H. (2008). Pharmacotherapy a pathophysiologic approach. In J. T. Dipiro, R. L. Talbert, G. C. Yee, G. R. Matzke, B. G. Wells, \& L. M. Posey (Eds.), Pharmacotherapy A Pathophysiologic Approach (7th ed., pp. 173-212). The McGraw-Hill Companies, Inc. https://doi.org/10.1036/007147899X

Ponikowski, P., Voors, A. A., Anker, S. D., Bueno, H., Cleland, J. G. F., Coats, A. J. S., Falk, V., Gonzalez-Juanatey, J. R., Harjola, V.-P., Jankowska, E. A., Jessup, M., Linde, C., Nihoyannopoulos, P., Parissis, J. T., Pieske, B., Riley, J. P., Rosano, G. M. C., Ruilope, L. M., Ruschitzka, F., ... Meer, P. van der. (2016). 2016 ESC Guidelines for the diagnosis and treatment of acute and chronic heart failure: The Task Force for the diagnosis and treatment of acute and chronic heart failure of the European Society of Cardiology (ESC). European Heart Journal, 1-85. https://doi.org/10.1093/eurheartj/ehw128

Rascati, K. (2014). Essentials of pharmacoeconomics (2nd ed.). Lippincott Williams \& Wilkins.

Syahidah, H. (2017). Kualitas hidup pasien kanker yang menjalani kemoterapi di RSI Sultan Agung Semarang. Universitas Muhammadiyah.

Tangsatitkiat, W., \& Sakthong, P. (2010). Thai version of the minnesota living with heart failure questionnaire: psychometric testing using a longitudinal design. Asian Biomedicine, 4(6), 877-884. https://doi.org/10.2478/abm-2010-0115

Teng, T. K., Tromp, J., Tay, W. T., Anand, I., Ouwerkerk, W., Chopra, V., Wander, G. S., Yap, J. J. L., MacDonald, M. R., Xu, C. F., Chia, Y. M., Shimizu, W., Richards, A. M., Voors, A., \& Lam, C. S. (2018). Prescribing patterns of evidence-based heart failure pharmacotherapy and outcomes in the ASIAN-HF registry: a cohort study. The Lancet Global Health, 6, e1008-e1018. https://doi.org/10.1016/S2214-109X(18)30306-1

Upadhya, B., \& Kitzman, D. W. (2017). Heart failure with preserved ejection fraction in older adult. Heart Failure Clinics, 13(3), 485-502. https://doi.org/10.1016/j.hfc.2017.02.005

WHO. (2007). Prevention of cardiovascular disease: pocket guidelines for assessment and management of cardiovascular risk. In World Health Organization. WHO Press.

WHO. (2020). Cardiovascular Diseases. World Health Organization.

Yaghoubi, A., Tabrizi, J., Mirinazhad, M., Azami, S., Naghavi-behzad, M., \& Ghojazadeh, M. (2012). Quality of life in cardiovascular patients in Iran and factors affecting It: a systematic Review. Journal of Cardiovascular and Thoracic Research, 4(4), 95-101. https://doi.org/10.5681/jcvtr.2012.024 